\title{
U-shaped Patterns in HRV From a Polysomnographic Point of View: a Quantitative Analysis
}

\author{
Mateusz Soliński ${ }^{1}$, Paweł Kuklik ${ }^{2}$, Jan Żebrowski ${ }^{1}$ \\ ${ }^{1}$ Faculty of Physics, Warsaw University of Technology \\ ${ }^{2}$ Department of Cardiology, Asklepios Hospital St. Georg, Hamburg, Germany, Faculty of Medicine, \\ Semmelweis University Campus Hamburg, Hamburg, Germany
}

\begin{abstract}
U-shaped patterns are acceleration-deceleration periods in RR interval series. These relatively short time events (average duration $29.8 \pm 4.1$ s) are the most laminar structures in the night-time recordings. The aim of this study is a analysis of sleep events occurring during $U$-shaped patterns in polysomnography (PSG) recordings obtained from Sleep Heart Health Study database. 500 PSG recordings were analyzed. U-shaped patterns were detected and categorized based on sleep stages, body position, respiratory events and EEG arousals. 4202 $U$-shaped patterns were found in 463 recordings. The majority of $U$-shaped patterns coincide with EEG arousals (73.9\%). $48 \%$ of the patterns occurred at sleep phase changes. Most of the U-shaped patterns were associated with the Wake phase (61\%). U-shaped patterns occurred at the position changes in $22.6 \%$ of cases. Analysis of respiratory events showed that $U$ shaped patterns occurred during hypopnea in $32.3 \%$ of the cases, CSA - 1.7\%, OSA - 0.8\% and desaturation $18.6 \%$. The quantitative analysis of PSG recordings is a first step to discover the origin of the phenomenon of $U$ shaped patterns. These first observations show that the $U$ shaped patterns are strongly associated with EEG arousals and may play role in sleep regulation.
\end{abstract}

\section{Introduction}

U-shaped patterns are characteristic changes of the heart rate observed during sleep. They are, in a domain of RR time intervals, the most laminar structures in nightrime recordings. They are defined as accelerationdeceleration periods in RR interval series, with duration between 20-40 s, and amplitude at smaller at least than $85 \%$ of the mean RR interval $[1,2]$.

U-shaped patterns were widely observed in healthy humans. Previous studies showed that these patterns have a considerable impact on the HRV parameters describing the VLF component, persistency, nonlinear correlations and multifractal properties, although their percentage contribution is small compared to the whole night-time series (on the average $3.1 \pm 1.7 \%$ ) $[1,3]$.

It is not completely clear which physiological mechanisms trigger U-shaped patterns in humans. Yazdani et al. using polysomnography recordings reported moderate correlation with movements during sleep [2]. In the literature we found many examples of acceleration-deceleration periods of RR time intervals during sleep associated with the sighs, leg movements, sleep apnea (i.e. cyclical variations of heart rate), arousals [4-7]; however, in most cases these changes of heart rate were too short and with smaller amplitude in comparison to U-shaped patterns. The phenomenon is different from the well-known HRV asymmetry observed by Porta et al [8]. This study is our first attempt to quantitative analysis of sleep events occurring during U-shaped patterns and investigate the origins of this phenomenon using polysomnography data.

\section{Data}

We use 500 polysomnography recordings (244 males; 59.7(10.7) years and 256 females; 60.6(10.3) years) from Sleep Heart Health Study (SHHS) database $[9,10]$ which is a part of The National Sleep Research Resource collection. The SHHS study was implemented by the National Heart Lung \& Blood Institute to determine the cardiovascular and other consequences of sleepdisordered breathing (ClinicalTrials.gov Identifier: NCT00005275). In selected group, 142 of patients had hypertension, 110 reported usually runny and snuffy nose, 101 frequent sinus infection, 21 diabetes, 38 asthma, 20 myocardial infraction and single cases of other cardiovascular and respiratory diseases.

Unattended polysomnography recordings were performed in home using The Compumedics P-Series Sleep Monitoring System. The system recorded the collection of signals: $\mathrm{SpO}_{2}$, heart rate (HR), EEG (C3-A2 and C4-A1), ECG (one channel), EOG (left and right), EMG, Respitrace Inducatnce Plethysmography 
(Thorax and Abdomen), Body position, external ambient light and nasal airflow. Sleep stages will be identified for each 30 second epoch using Rechtshaffen and Kales criteria [11]. EEG arousals and breath-related events such as: hypopneas (identified if the amplitude of any respiratory signal is reduced by $30 \%$ of the amplitude of "baseline", if this change lasts for $\geq 10 \mathrm{~s}$ and for $>2$ breaths), $\mathrm{SpO}_{2}$ desaturation, obstructive and central sleep apnea (OSA, CSA) were annotated using standard criteria.

\section{Methods}

U-shaped patterns were detected from night-time RR intervals series using automatic algorithm based on support vector machines. Next, the number and the percentage of total detected U-shaped patterns coexistence with the sleep events were calculated. The coexistence with the pattern was annotated when the sleep event begun or ended in any moment of occurring of the U-shaped pattern.

\section{Results}

The results descriptive analysis of total number of U-shaped patterns detected in the study group were presented in Table 1. U-shaped patterns were found in $92.6 \%$ of patients and mean number of U-shaped patterns was $8.4(7.7)$ per patient. We observed that the number of U-shaped patterns increasing with the sleep time. The histogram of probability density of occurring U-shaped patterns in the following hours before wake up was showed in Figure 1.

The most common case of the coexistence of U-shaped patterns and the other sleep events was presented in Figure 2. In this example U-shaped patterns occurred at the EEG arousal, wake sleep stage, and excitations in EMG, EEG, Thorax and Abdomen signals, but with no change in $\mathrm{SpO}_{2}$ level.

Table 1. Descriptive analysis of the number of U-shaped patterns and their properties in the study group.

\begin{tabular}{lc}
\hline $\begin{array}{l}\text { Total number of U-shaped } \\
\text { patterns }\end{array}$ & $\mathbf{4 2 0 2}$ \\
\hline Mean per patient & $8.4(7.7)$ \\
\hline Min-max & $0-47$ \\
\hline $\begin{array}{l}\text { Number of patients with at } \\
\text { least one U-shaped pattern }\end{array}$ & $463 / 500(92.6 \%)$ \\
\hline Mean length & $44(12)$ RR intervals \\
\hline $\begin{array}{l}\text { Mean relative amplitude } \\
\text { (related to mean RR interval) }\end{array}$ & $66.9(15.3) \%$ \\
\hline
\end{tabular}

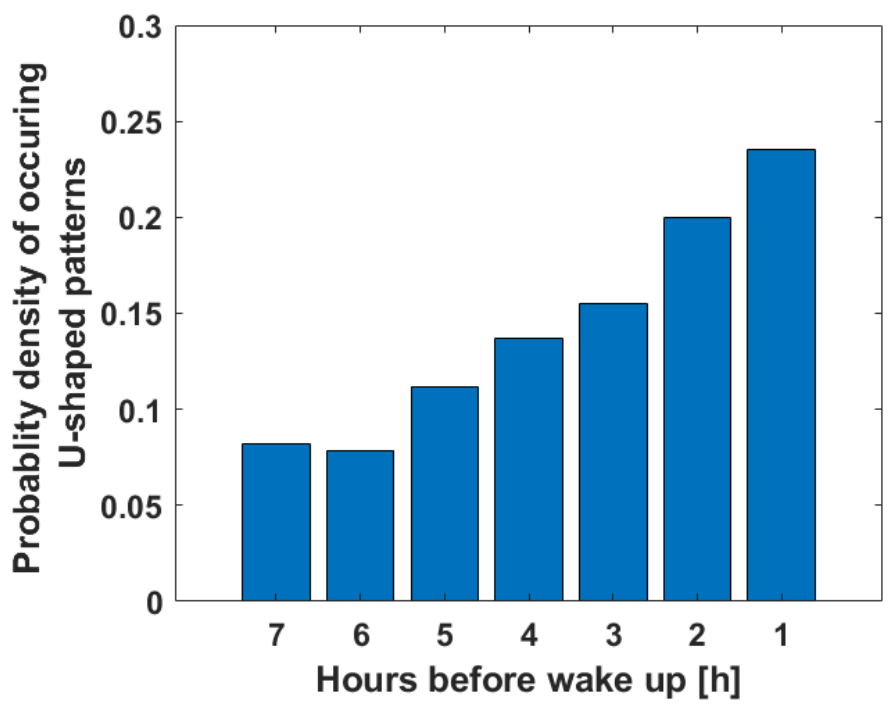

Figure 1. Probability density of the occurrence of the Ushaped patterns in the last $7 \mathrm{~h}$ of sleep.

U-shaped patterns occurred with the wake stage in $27.5 \%$ cases, $12.1 \%$ during N2, $11.0 \%$ during REM, $0.7 \%$ during $\mathrm{N} 3$ and $0.7 \%$ during $\mathrm{N} 1$. The rest $48 \%$ of U-shaped patterns occurred at sleep stage changes $(33.4 \%$ of the patterns occurred at sleep stage changes from or to wake stage). The percentage distribution of the sleep stages in a study group with at least one detected U-shaped pattern was: $\mathrm{N} 2-43 \%, \mathrm{~W}-24 \%, \mathrm{REM}-16 \%$, $\mathrm{N} 3-12 \%, \mathrm{~N} 1-4 \%$ and $\mathrm{N} 4-1 \%$.

Significantly more U-shaped patterns were detected at supine and prone body position, $25.6 \%$ and $22.4 \%$ of total number of the patterns, respectively, in comparison to back positions $-15.2 \%$ at right and $14.3 \%$ at left. $22.5 \%$ of all detected U-shaped patterns occurred during body position changes. The percentage distribution of the body position in a study group with at least one detected U-shaped pattern was: supine $-32 \%$, prone $-22 \%$, right $-23 \%$ and left $-23 \%$.

We observed high coexistence level between U-shaped patterns and EEG arousals. $73.9 \%$ of all detected patterns occurred at these events. Considering breath-related events, $32.3 \%$ of all U-shaped patterns occurred during hypopnea, $18.6 \%$ during $\mathrm{SpO}_{2}$ desaturation and only $0.8 \%$ and $1.7 \%$ with OSA and CSA, respectively. The percentage of U-shaped patterns occurred at EEG arousals and breath-related events are showed in Table 2. 


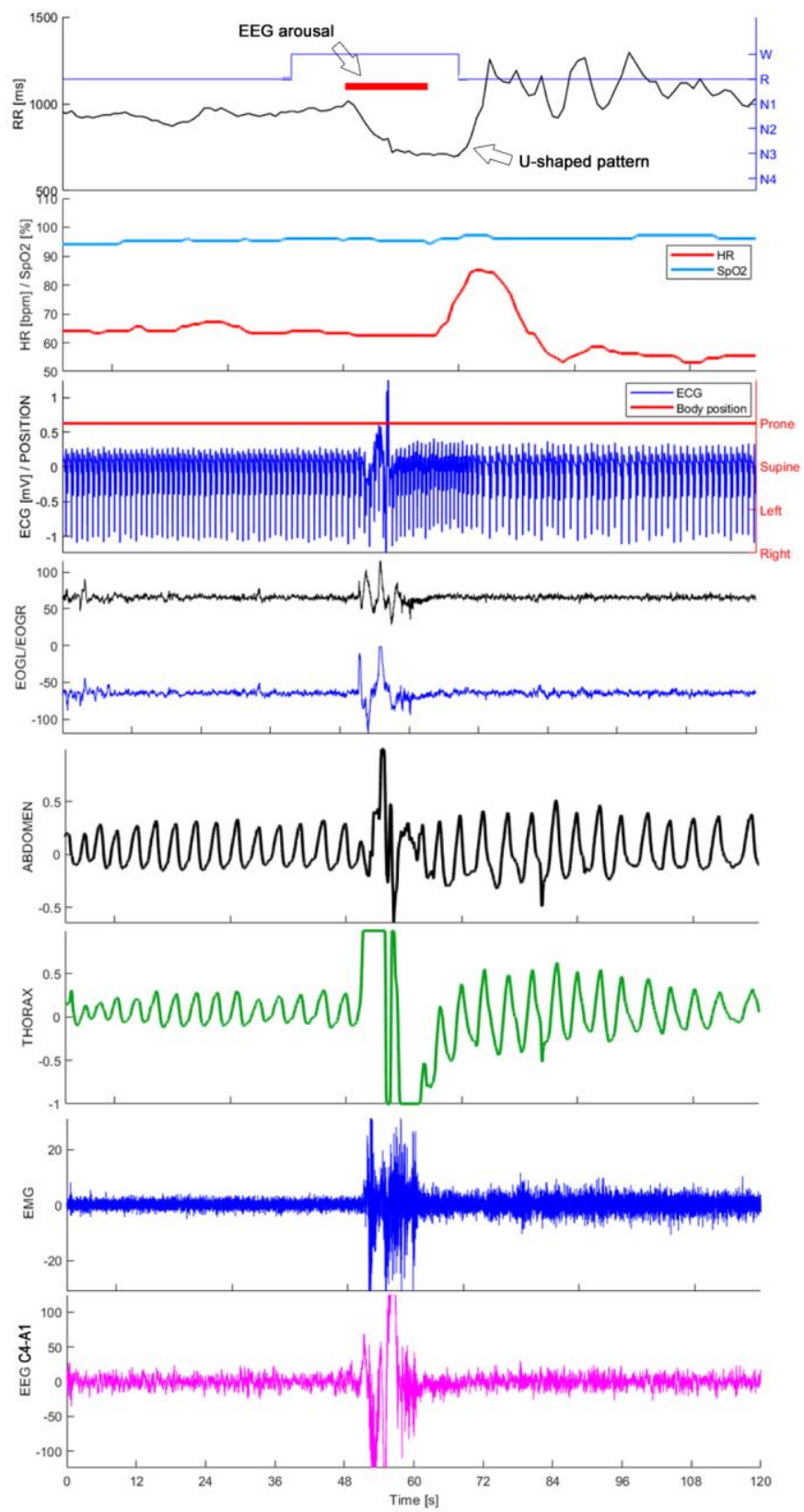

Figure 2. Example of U-shaped pattern, sleep events and the signals obtained from unattended polysomnography recording.

\section{Discussion}

Low level of coexistence between the U-shaped patterns and body position changes, OSA, CSA enhances prior assumptions, based on literature findings, that the main origin of U-shaped patterns are not movements and apneas (unfortunately, in SHHS database there are not signals from leg movements). At once, the quantitative analysis showed that the largest number of U-shaped patterns occurred at Wake sleep stages $(27.5 \%$ of all
U-shaped patterns), sleep stage changes from or to Wake stage $(33.4 \%)$ and EEG arousals (73.9\%). 1738/4202 (41.4\%) of U-shaped patterns occurred during either Wake stage (or sleep stage changes from or to Wake stage) and EEG arousal (according to arousals classification criteria, brief arousals (e.g. arousals $<15 \mathrm{~s}$. long) do not automatically require a change in sleep stage).

Table 2. Percentage of U-shaped patterns occurred at sleep events.

\begin{tabular}{ccc}
\hline Sleep event & $\begin{array}{c}\text { Total number } \\
\text { of events in } \\
\text { database }\end{array}$ & $\begin{array}{c}\% \text { of U-shaped } \\
\text { patterns occurred at } \\
\text { the sleep event }\end{array}$ \\
\hline EEG arousal & 51077 & $73.9 \%(3105 / 4202)$ \\
\hline Hypopnea & 92823 & $32.3 \%(1357 / 4202)$ \\
\hline $\mathrm{SpO}_{2}$ desaturation & 61880 & $18.6 \%(780 / 4202)$ \\
\hline OSA & 5081 & $0.8 \%(34 / 4202)$ \\
\hline CSA & 1646 & $1.7 \%(71 / 4202)$ \\
\hline
\end{tabular}

Arousals are divided in 4 levels, including D-bursts and K-bursts (subcortical arousals), the standard definition of a microarousal (MA) and phases of transitory activation (PAT; MA and PAT are considered as cortical arousals) [12]. We found similarities between U-shaped patterns and heart rate responses to PAT arousals including duration, mean number at night (per patient) and distribution during sleep [12, 13]. The examples of cardiac responses to PAT arousals, similar to the U-shaped patterns, was found in the study by Basner et al. [14] as well.

EEG arousals play a regulatory role in the sleep. It was shown that cardiac responses due to these sleep events are initialized by sympathetic activation [12]. The number of arousals rely on sleep propensity and sleep pressure. At the beginning of the night, when the sleep pressure is high, the arousal threshold is relatively high and peaking of subcortical D-bursts and K-bursts, characterized by lower cardiac responses, is present. Alternatively, at the end of the sleep, the arousal threshold is lower and the number of cortical (PAT) arousals (with much stronger cardiac responses) increases. It is complementary with the probability density of U-shaped patterns observed in this and our previous studies. Following this hypothesis, U-shaped patterns, as the cardiac response of cortical arousals (PAT), may be considered as a marker of sleep regulation. It is also supported by Yazdani et al. study which showed that the features of U-shaped patterns change due to sleep deprivation [15]. 


\section{Conclusions}

In this study, we showed that U-shaped patterns occur with sleep-related events observed in polysomnography recordings. We found that $\mathrm{U}$-shaped patterns are associated with EEG arousals in much greater degree than other sleep events. We indicated similarities between cardiac responses (initialized by sympathetic activation) due to PAT arousals and U-shaped patterns. These observations suggest that these patterns may play the role in sleep regulation.

\section{Acknowledgments}

We would like to thank our colleagues from the Cardiovascular Physics Group at the Faculty of Physics of Warsaw University of Technology for support.

The Sleep Heart Health Study (SHHS) was supported by National Heart, Lung, and Blood Institute cooperative agreements U01HL53916 (University of California, Davis), U01HL53931 (New York University), U01HL53934 (University of Minnesota), U01HL53937 and U01HL64360 (Johns Hopkins University), U01HL53938 (University of Arizona), U01HL53940 (University of Washington), U01HL53941 (Boston University), and U01HL63463 (Case Western Reserve University). The National Sleep Research Resource was supported by the National Heart, Lung, and Blood Institute (R24 HL114473, RFP 75N92019R002).

\section{References}

[1] Soliński M, Kuklik P, Gierałtowski JJ, Baranowski R, Graff B, Żebrowski JJ. "The effect of persistent Ushaped patterns in RR night-time series on the heart rate variability complexity in healthy humans", Physiological Measurement, Vol. 41, No. 6, May 2020, Art. no. 065001.

[2] Yazdani S, Cherqui A, Bourdillon N, Millet G, Vesin JM, "Sleep RR-Interval U-patterns and their correlation to movement events", Computing in Cardiology (CinC), Vol. 46, Sep. 2019, pp. 1-4.

[3] Soliński M, Baranowski R, Graff B, Żebrowski J., "The effect of U-Shaped patterns to nonlinear properties of heart rate variability", Computing in Cardiology (CinC), Vol. 46, Sep. 2019, pp. 1-4.

[4] Perez-Padilla R, West P, Kryger MH, "Sighs during sleep in adult humans", Sleep, Vol. 6, No. 3, Sep. 1983, pp. 234-43.

[5] Winkelman JW, "The evoked heart rate response to periodic leg movements of sleep", Sleep, Vol. 22, No. 5, Aug. 1999, pp. 575-80.

[6] Baumert M, Kohler M, Kabir M, Sanders P, Kennedy
D, Martin J, Pamula Y, "Altered cardio-respiratory response to spontaneous cortical arousals in children with upper airway obstruction", Sleep Medicine, Vol. 12, No. 3, Mar. 2011, pp. 230-8.

[7] Bonnet MH, Arand DL, "Heart rate variability: sleep stage, time of night, and arousal influences", Clin. Neurophysiol. May 1997, Vol. 102, No. 5, pp. 390-6.

[8] Porta A, Guzzetti S, Montano N, Gnecchi-Ruscone T, Furlan R, Malliani A, "Time reversibility in shortterm heart period variability", Computing in Cardiology (CinC), Vol. 33, Sep. 2006, pp. 77-80.

[9] Zhang GQ, Cui L, Mueller R, Tao S, Kim M, Rueschman M, Mariani S, Mobley D, Redline S, "The National Sleep Research Resource: towards a sleep data commons", J Am Med Inform Assoc, Vol. 25, No. 10, Oct. 2018, pp. 1351-8.

[10] Quan SF, Howard BV, Iber C, Kiley JP, Nieto FJ, O'Connor GT, Rapoport DM, Redline S, Robbins J, Samet JM, Wahl PW, "The sleep heart health study: design, rationale, and methods", Sleep, Vol. 20, No. 23, Dec. 1997, pp. 1077-85.

[11] Rechtshaffen A, Kales A, "A manual of standardized terminology techniques and scoring system for sleep stages in human subjects", Government Printing Office, Washington, DC: US, 1968.

[12] Sforza E, Jouny C, Ibanez V, "Cardiac activation during arousal in humans: further evidence for hierarchy in the arousal response", Clin Neurophysiol, Vol. 111, No. 9, Sep. 2000, pp. 1611-9.

[13] Sforza E, Chapotot F, Pigeau R, Buguet A, "Time of night and first night effects on arousal response in healthy adults", Clin Neurophysiol, Vol. 119, No. 7, Jul. 2008, pp. 1590-9.

[14] Basner M, Griefahn B, Müller U, Plath G, Samel A, "An ECG-based algorithm for the automatic identification of autonomic activations associated with cortical arousal", Sleep, Vol. 30, No. 10, Oct. 2007, pp. 1349-61.

[15] Yazdani S, Cherqui A, Bourdillon N, Millet G, Vesin JM, "Analysis of U-shape patterns in RR-interval time series during sleep", Computing in Cardiology (CinC), Vol. 45, Sep. 2019, pp. 1-4.

Address for correspondence:

Mateusz Soliński, Koszykowa 75 St. Warsaw 00-662 Poland, mateusz.solinski.dokt@pw.edu.pl 